\title{
Inulina: una alternativa para el desarrollo de productos cárnicos funcionales
}

\author{
María Alicia Peña ${ }^{1}$, Silvia Peña ${ }^{2,3}$, María Aloida Guerra ${ }^{4}$ \\ 'Facultad de Ciencia y Tecnología, Universidad del Azuay, Av. 24 de Mayo 7-77 y Hernán Malo, \\ Cuenca, Ecuador \\ ${ }^{2}$ Facultad de Ciencias Químicas, Universidad de Cuenca, Av. 12 de Abril, Cuenca, Ecuador \\ 32Departamento de Engenharia de Produção, Universidade Federal de Minas Gerais, Av. Pres. \\ Antônio Carlos, 6627 - Pampulha, Belo Horizonte - MG, 31270-901, Brasil \\ ${ }^{4}$ Instituto de Investigaciones Científicas para la Industria Alimenticia (IIIA), Carretera al Guatao km \\ 31/2, La Habana, Cuba. \\ *Autor para correspondencia / Corresponding author, email: mpenag@uazuay.edu.ec
}

\section{Inulin: an alternative for the development of functional meat products}

\section{RESUMEN}

Los alimentos funcionales actualmente tienen una alta demanda en el mercado, lo cual constituye un desafí importante para la industria alimentaria. Es por ello, que el objetivo del presente trabajo fue elaborar un producto cárnico funcional con la adición de inulina logrando sustituir grasa y obtener un efecto prebiótico. Para ello se realizaron experimentos con combinaciones de inulina (0 a 12\%) y grasa (8 a 12\%), empleando almidón de papa al 4\% en las formulaciones. A fin de evaluar las salchichas obtenidas en cada experimento se determinó: composición fisicoquímica, análisis de perfil textura, análisis microbiológicos y evaluación sensorial. Se caracterizó el material de envase y se determinó la vida útil de las salchichas seleccionadas envasadas al vacío, estudiando dos tratamientos posteriores: uno refrigeradas y el otro repasteurizadas-refrigeradas manteniendo en ambos casos una temperatura de $2-4^{\circ} \mathrm{C}$. Las muestras se caracterizaron al inicio y final del ensayo desde el punto de vista fisicoquímico y reológico, durante todo el estudio se realizaron análisis microbiológicos y evaluación sensorial, lo que permitió determinar la vida útil a través de un criterio de aceptación y rechazo. Tomando como base criterios reológicos y sensoriales, la combinación con un 6.67\% de inulina y 8,73\% de grasa resultó ser la mejor variante. Se observó que la inulina produce un aumento significativo de la dureza de los productos, sin embargo, no afecta las características sensoriales y mantiene la jugosidad del producto. El criterio de evaluación sensorial utilizado determinó una vida útil de 26 días para las con tratamiento envasadas al vacío refrigeradas y 112 días para las muestras repasteurizadas-refrigeradas mediante el gráfico de riesgos de Weibull. Resultados que ratifican la técnica de repasteurización como una opción viable para uso industrial.

Palabras claves: alimento funcional, grasa, inulina, salchicha, vida útil. 


\begin{abstract}
Functional foods are currently in high demand in the market, which is an important challenge for the food industry. Thus, the objective of this work was to develop a functional meat product with the addition of inulin, replacing fat and obtaining a prebiotic effect. For this, experiment several combinations of inulin and fat were used: inulin (0 to 12\%) and fat (8 to 12\%), using potato starch $4 \%$ as an ingredient in the formulations. For each sausage physicochemical composition, texture profile analysis, sensory evaluation and microbiological analyses were carried out. The packaging material was characterized and the shelf life was determined for the sausages by studying two different treatments: a vacuum packed, refrigerated product and the other a vacuum packed, re-pasteurized, refrigerated product in both cases maintaining a temperature of $2-4^{\circ} \mathrm{C}$. The samples were studied at the beginning and the end of the experiment by physicochemical analysis, rheological characterization, throughout the study microbiological analysis and sensory evaluation were carried out. For the analysis of shelf life, acceptance and rejection criteria were used. Taking as reference rheological and sensory criteria, the combination with $6,67 \%$ inulin and $8,87 \%$ fat proved to be the best variant. It was observed that inulin produces a significant increase in the hardness of the products, however, it does not affect the sensory characteristics and maintains the juiciness of the product. The sensory evaluation criteria have been used to determinate the shelf life, resulting in 26 days for the vacuum-packed refrigerated sausages, while the vacuum packed re-pasteurized refrigerated sausages lasted 112 days, results obtained by Weibull plot. As a conclusion of this research, the repasteurization technique can be a viable process for industrial use.
\end{abstract}

Keywords: functional food, fat, inulin, sausage, shelf life.

\title{
INTRODUCCIÓN
}

Dada la estrecha relación existente entre la dieta y la salud, se ha evidenciado una creciente preocupación de los consumidores por alimentos saludables, lo que ha dado lugar a la aparición y crecimiento exponencial de nuevas tendencias de consumo de los llamados alimentos funcionales. Estos productos se refieren a los alimentos procesados que contienen ingredientes que desempeñan una función específica en la fisiología del organismo humano, más allá de su contenido nutrimental [1].

Por lo antes expuesto, en el sector alimentario han surgido diferentes alternativas de ingredientes funcionales, tal es el caso de la inulina. Este carbohidrato posee un efecto prebiótico que estimula el crecimiento selectivo y/o la actividad metabólica de un número limitado de bacterias en el colón [2]. Los prebióticos promueven la flora intestinal natural proporcionando nutrientes para las bacterias benéficas (bifidobacterias y lactobacilos) existentes, con la consecuente disminución de otras especies que pueden ser perjudiciales como: Escherichia coli, Clostridium spp, entre otros [3].

Se trata de un carbohidrato no digerible que se encuentra en frutas, vegetales y cereales, que en forma nativa es una mezcla de oligómeros y polímeros con un número variable 
de moléculas de fructosa, unidas por enlaces $\beta$-(2-1) que pueden incluir en su extremo una molécula de glucosa [4]. Posee la poderosa propiedad de sustituir grasa a partir de su capacidad de gelificación con agua bajo fuerza de corte. El gel resultante tiene una textura cremosa que mejora la estabilidad de emulsiones, muestra excepcionales características semejantes a la grasa y presenta un efecto sinérgico con agentes gelificantes [3].

Por otro lado, los productos cárnicos son parte fundamental en una dieta diaria, pues aportan nutrientes de gran importancia para el desarrollo humano como ciertas vitaminas (siendo la fuente más importante la vitamina $\mathrm{B}_{12}$ ), ácidos grasos y minerales (hierro y zinc) [5]. Su importancia nutricional radica principalmente en su importante contenido proteico de alto valor biológico. Sin embargo, los derivados cárnicos pueden contener sustancias que, al ser añadidas o formadas durante su procesamiento, conservación o consumo, en proporciones inadecuadas, pueden afectar adversamente la salud de los consumidores [6].

Específicamente, el alto contenido de sodio y grasa son los principales factores de riesgo asociados al consumo de productos cárnicos [7]. Diferentes estudios reportan que existe una alta probabilidad de padecer alguna de las enfermedades crónicas no trasmisibles por una dieta con elevados índices de grasas saturadas, colesterol y sal. En los últimos años diferentes estudios recogen evidencias sobre los riesgos del consumo de carnes rojas y la enfermedad cardiovascular (ECV), el cáncer, obesidad y diabetes tipo 2 [5].

Con el objetivo de reducir el contenido de grasa en derivados cárnicos una gran variedad de métodos ha sido desarrollada. Dentro de estas alternativas se destaca el uso de ingredientes no cárnicos como derivados proteicos de origen vegetal y carbohidratos, los cuales han sido utilizados en la elaboración de embutidos con propósitos tecnológicos como: disminuir el contenido de grasa, bajar costos de formulación e incrementar su valor nutritivo [8]. Los sustitutos de grasa a base de carbohidratos tienen la ventaja de imitarla, otorgando características similares en términos de lubricidad y humedad [9].

En este contexto, el objetivo del presente trabajo es establecer el potencial efecto que presenta la adición de inulina, en la elaboración de productos cárnicos emulsificados funcionales, con bajo contenido en grasa y que cumplan con los requisitos nutricionales y de calidad.

\section{MATERIALES Y MÉTODOS}

Para el desarrollo del derivado cárnico emulsificado (salchicha) con propiedades funcionales se empleó carne de cerdo con un contenido de grasa del 5\% y tocino de lomo. Estas materias primas se obtuvieron de cerdos con 48 horas de almacenamiento refrigerado post-mortem.

Como ingredientes no cárnicos se utilizó inulina (Orafti, Chile) y almidón de papa (Fai Berti S.L., España), por sus conocidas propiedades funcionales y nutricionales, actuando sobre las pérdidas de agua en la cocción y la estabilidad de la emulsión [10-12]. Además, se utilizó una solución de colorante carmín, sales, condimentos y humo líquido. 
Fue empleado un diseño factorial $3^{2}$, variando la cantidad de inulina: $X_{1}(0$ a $12 \%)$ y grasa: $X_{2}$ (8 a 12\%). El resto de los componentes se mantuvieron constantes. Los valores máximos y mínimos de inulina y tocino de lomo y el porcentaje de almidón de papa fueron seleccionados en función de los resultados obtenidos en pruebas de observación y reportes de la literatura [13-15]. De este procedimiento se definieron nueve combinaciones experimentales (Tabla 1). Se elaboraron 4 kg de salchichas de acuerdo a cada combinación experimental. De cada una de las variables se procesaron tres corridas experimentales.

TABLA 1. Puntos experimentales (Diseño factorial).

\begin{tabular}{|c|c|c|c|c|c|c|c|c|c|c|}
\hline Variantes & & $\mathbf{1}$ & $\mathbf{2}$ & $\mathbf{3}$ & $\mathbf{4}$ & $\mathbf{5}$ & $\mathbf{6}$ & $\mathbf{7}$ & $\mathbf{8}$ & $\mathbf{9}$ \\
\hline Inulina (\%) & & 0 & 6 & 1 & 0 & 6 & 1 & 0 & 6 & 1 \\
\hline Tocino de lomo (\%) & & & & 2 & & & 2 & & \\
\hline
\end{tabular}

El proceso de elaboración fue el mismo que se usa habitualmente para la elaboración de la salchicha tradicional. Se prepararon piezas de $45 \mathrm{~g}$ empleando tripas impermeables de poliamida 6 (Kalle Nalo, España) de 22 mm de diámetro, y se envasaron en bolsas al vacío. La carne fue caracterizada mediante las determinaciones descritas en la Tabla 2:

TABLA 2. Determinaciones físico-químicas de la carne

\begin{tabular}{|c|c|}
\hline Parámetro & Método \\
\hline Humedad & NC 275 [16] \\
\hline Grasa & NC ISO $1443[17]$ \\
\hline Proteína & NC ISO $937[18]$ \\
\hline pH & NC ISO 2917 [19] \\
\hline
\end{tabular}

Para caracterizar la inulina se emplearon ensayos físico-químicos como: humedad [20], ceniza [22] y pH [22], así como la fuerza de gel, para lo cual se prepararon dos soluciones: 1) inulina al 40\% en agua destilada y 2) solución con $40 \%$ de inulina y $4 \%$ de almidón de papa en agua destilada. Cada una de las soluciones se calentó hasta $80{ }^{\circ} \mathrm{C}$ por 3 minutos con agitación constante, se enfrío hasta $10{ }^{\circ} \mathrm{C}$ y posteriormente se refrigeró entre 4 a $6{ }^{\circ} \mathrm{C}$ por 24 horas, lapso de tiempo en el que se alcanza la máxima fuerza de gel [23]. De los geles obtenidos se determinó su fuerza mediante el analizador de textura T.A HD Plus a $20^{\circ} \mathrm{C}$, para ello la penetración se realizó con una sonda de $1,9 \mathrm{~cm}$ de diámetro a la velocidad de $1 \mathrm{~mm} / \mathrm{s}$ y del gráfico obtenido se calculó la fuerza de gel expresada en gramos, siendo el valor máximo de la curva [24]. Adicionalmente, se elaboró una salchicha control (tradicional) con un 60\% de carne de cerdo, un $25 \%$ de grasa y un $2 \%$ de proteína aislada de soya. En las salchichas obtenidas, se determinó la humedad, proteína, pH y grasa, mediante los métodos anteriormente reportados para carne, además de cenizas [25], cloruro de sodio [26] y nitrito de sodio [27].

Para los análisis microbiológicos, la toma de muestras fue llevada a cabo inmediatamente después del proceso de elaboración, realizado los ensayos descritos en la siguiente tabla: 


\section{TABLA 3. Determinaciones microbiológicas}

\begin{tabular}{|c|c|}
\hline Parámetro & Método \\
\hline Aerobios mesófilos & NC ISO 4833-1 [28] \\
\hline Conteo de coliformes fecales & NC ISO 4831 [29] \\
\hline Conteo de coliformes totales & NC ISO 4832 [30] \\
\hline Conteo de mohos y levaduras totales & NC 1004 [31] \\
\hline Salmonella & NC ISO 6579 [32] \\
\hline Staphylococcus coagulasa & NC ISO 6888-1 [33] \\
\hline
\end{tabular}

Adicionalmente se realizaron conteos de psicrófilos (en Análisis de Componentes Principales, $\mathrm{ACP}, 4$ a 7 días, 2 a $4^{\circ} \mathrm{C}$ ) y conteo de bacterias ácido lácticas (en medio de cultivo agar MRS, 24 $\mathrm{h}, 37^{\circ} \mathrm{C}$ ). La calidad sensorial fue evaluada por 12 jueces entrenados, empleando una escala de valoración de calidad estructurada de siete puntos (1: pésimo y 7: excelente) para los atributos aspecto, textura, sabor y color. Para la jugosidad se utilizó una escala de siete puntos no estructurada (1: extremadamente seca y 7: extremadamente jugosa).

Para el perfil de textura se aplicó una prueba de compresión doble con un texturómetro universal Instron, modelo 1140 (ITW, USA). Se comprimió diametralmente hasta un 75\% a una velocidad de $20 \mathrm{~cm} \cdot \mathrm{min}^{-1}$. A partir del gráfico fuerza-distancia, se determinaron las siguientes propiedades: dureza, elasticidad y cohesividad [24]. Se caracterizó el material de envase (tripas impermeables y bolsas para envasado al vacío), mediante un espectrofotómetro infrarrojo, modelo Vector 22 (Bruker, Suiza), en un rango de medición entre 4000-600 cm-1. Se determinaron las propiedades físico-mecánicas de: peso base [34], espesor [35] y resistencia a la tensión y elongación [36], así como a la permeabilidad al vapor de agua [37] y la resistencia del sellado térmico [38].

Para la selección de la mejor variante, se realizó la optimización en base al espacio de diseño acotado, para lo cual se impusieron restricciones (Tabla 4), que fueron fijadas en base a la formulación tradicional y criterios de diferentes especialistas [39]. Asimismo, se estableció priorizar el menor contenido de grasa con el objetivo de obtener un alimento funcional y económico.

TABLA 4. Restricciones impuestas al sistema para la optimización

\begin{tabular}{|c|c|}
\hline Variable Respuesta & Restricción \\
\hline Dureza instrumental & 5 a $8 \mathrm{~kg}$ \\
\hline Elasticidad instrumental & 6 a $8 \mathrm{~mm}$ \\
\hline Aspecto & $>5$ \\
\hline Textura & $>5$ \\
\hline Sabor & $>5$ \\
\hline Color & $>5$ \\
\hline Jugosidad & 3 a 4 \\
\hline (Siendo 1: pésima valoración y 7: excelente) & \\
\hline
\end{tabular}


Los atributos sensoriales y los parámetros de perfil de textura se procesaron mediante el programa "Design Expert Analysis" versión 7 para ajustar los modelos, generar las ecuaciones y sus correspondientes superficies de respuesta. El análisis estadístico de estos resultados se realizó mediante el programa Statgraphics Centurion XVI. A los resultados fisicoquímicos y microbiológicos de los productos, se les determinó la media y la desviación estándar.

Para el estudio de vida útil se tomó la variante seleccionada, se procesaron nuevos lotes de salchichas y se realizaron dos tratamientos de conservación: refrigeración $\left(2-4^{\circ} \mathrm{C}\right)$ y repasteurización (baño maría a $80^{\circ} \mathrm{C} / 10 \mathrm{~min}$ ) seguida de refrigeración $\left(2-4^{\circ} \mathrm{C}\right)$. Durante este estudio se realizaron análisis fisicoquímicos, microbiológicos, evaluación sensorial y de textura instrumental al inicio del estudio y en el momento de rechazo.

Para determinar la vida útil del producto se aplicó el gráfico de riesgos de Weibull, utilizando un intervalo de confianza del 95\% [40].

\section{RESULTADOS Y DISCUSIÓN}

La Tabla 5 muestra los contenidos de humedad, ceniza y valor del pH de la inulina. Los valores obtenidos se asemejan con lo reportado por la literatura [41]. En el caso de las cenizas $(0,45 \%)$ está fuera del valor señalado por la literatura $(<0,2 \%)$ sin embargo está dentro del rango reportado por el fabricante.

TABLA 5. Composición físico-química de la de la inulina

\begin{tabular}{|c|c|c|}
\hline Humedad (\%) & Ceniza (\%) & $\mathrm{pH}$ \\
\hline $4,85(0,02)^{*}$ & $0,45(0,01)^{*}$ & 6,0 \\
\hline
\end{tabular}

Los resultados obtenidos de la fuerza de gel de las soluciones ensayadas se muestran en la Tabla 6. Al comparar estos resultados con los reportados por Kim y col. (2001) quienes determinaron la fuerza de gel en la inulina Ratilin HP de la marca Orafti, se observa que los valores alcanzados en sus ensayos son mucho mayores en relación a los obtenidos en este estudio. Esta diferencia puede ser atribuida a que la inulina que se utilizó en la presente investigación es de un grado de polimerización más bajo (no reportado por el fabricante) al utilizado en el estudio citado, pues la literatura señala que mientras mayor sea el grado de polimerización de la inulina se obtienen geles más firmes [42]. En la misma tabla, se puede apreciar, que la presencia de almidón de papa (4\%) favoreció la formación de gel de la inulina, obteniéndose una fuerza de gel mayor $(82,73 \mathrm{~g})$, lo cual demuestra el efecto sinérgico entre estos dos elementos. Esto concuerda con lo descrito por Madrigal (2007) [41] quien describe un efecto sinérgico entre la inulina y otros agentes gelantes. 


\section{TABLA 6. Propiedades funcionales de la inulina}

\begin{tabular}{|c|c|}
\hline Solución & Fuerza de gel (g) \\
\hline Inulina al 40\% & $36.93(2.05)$ \\
\hline Inulina al 40\% + almidón de papa al 4\% & $82.73(4.26)$ \\
\hline
\end{tabular}

*( ) Desviación Estándar, $n=3$

Los resultados de los análisis de composición química y pH de las formulaciones propuestas en el diseño se reflejan en la Tabla 7. En los valores obtenidos para la humedad se observa una ligera tendencia de disminución de este parámetro a medida que aumenta la concentración de inulina. Resultados que son similares a los especificados en la literatura para productos con bajo contenido en grasa y altos niveles de agua añadidos [43]. La variación en la humedad puede deberse tanto a la presencia de inulina y almidón de papa, como al contenido en grasa, ya que, en las condiciones estudiadas, la disminución del nivel de grasa se realizó a costa de aumentar la cantidad de agua añadida.

TABLA 7. Composición proximal de las salchichas experimentales.

\begin{tabular}{|c|c|c|c|c|c|c|c|c|}
\hline 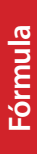 & 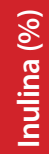 & $\frac{2}{2}$ & 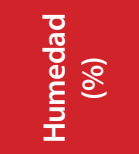 & $\begin{array}{c}\frac{2}{2} \\
\frac{8}{4} \\
\frac{0}{0}\end{array}$ & 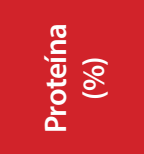 & $\frac{\sqrt{0}}{\frac{\pi}{2}}$ & 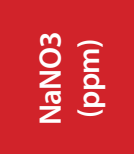 & I \\
\hline 1 & 0 & 8 & $69,79(0,05)$ & $8,50(2,4)$ & $10,79(0,53)$ & $1,82(0,02)$ & $98,3(2,83)$ & 6,20 \\
\hline 2 & 6 & 8 & $67,50(0,23)$ & $7,33(2,74)$ & $11,00(0,76)$ & $2,07(0,02)$ & $73,2(0,65)$ & 6,40 \\
\hline 3 & 12 & 8 & $66,70(0,13)$ & $8,32(3,15)$ & $10,84(0,96)$ & $1,82(0,05)$ & $60,9(0,81)$ & 6,09 \\
\hline 4 & 0 & 10 & $64,14(0,16)$ & $9,94(1,65)$ & $10,91(0,83)$ & $1,82(0,09)$ & $54,4(3,75)$ & 6,17 \\
\hline 5 & 6 & 10 & $63,70(0,08)$ & $10,00(1,28)$ & $11,96(0,5)$ & $2,05(0,06)$ & $63,3(2,24)$ & 6,43 \\
\hline 6 & 12 & 10 & $61,36(0,13)$ & $10,45(3,62)$ & $12,00(1,23)$ & $1,84(0,08)$ & $84,4(0,92)$ & 6,12 \\
\hline 7 & 0 & 12 & $69,59(0,04)$ & $12,00(0,96)$ & $10,67(0,35)$ & $1,47(0,03)$ & $78,9(3,75)$ & 6,25 \\
\hline 8 & 6 & 12 & $64,52(0,47)$ & $12,23(1,62)$ & $11,15(0,77)$ & $2,07(0,06)$ & $67,9(2,87)$ & 6,40 \\
\hline 9 & 12 & 12 & $59,66(0,21)$ & $12,36(1,77)$ & $11,95(0,84)$ & $1,94(0,08)$ & $87,3(1,29)$ & 6,15 \\
\hline \multicolumn{3}{|c|}{ Control } & $58,48(0,02)$ & $24,00(2,36)$ & $12,59(1,56)$ & $1,62(0,03)$ & $79,76(0,01)$ & 6,2 \\
\hline
\end{tabular}

()* Desviación Estándar; $\mathrm{n}=3$

Las variaciones de grasa fluctúan en valores que van del 7 al 12\% que, si se compara con la salchicha control que tiene un $24 \%$ de grasa, se puede observar que se ha logrado una disminución considerable de hasta un 65\%, semejante a lo logrado en otros trabajos con adición de inulina [44-46]. Los valores de grasa corresponden con los niveles diseñados para las fórmulas utilizadas. En el valor de $\mathrm{pH}$ se observa que no hay diferencias significativas entre los diferentes experimentos, por lo que se asume que la 
inulina no tiene incidencia en este parámetro, concordando con los trabajos reportados por otros autores: $[47,48,45]$. Los contenidos de proteína obtenidos van desde 10,64\% hasta $12 \%$, al establecerse un contenido de carne constante en todos los casos, no se observan diferencias sustanciales.

Los resultados microbiológicos se pueden observar en la Figura 1, obteniéndose conteos de microorganismos mesófilos y psicrófilos bajos, manteniendo los niveles adecuados para este tipo de producto (menor a 1,0 × 106 UFC/g) según la norma NTE INEN 1338:2012 [50]. En el caso de las enterobacterias los conteos dieron resultados con valores inferiores a los límites de detección, lo que estuvo en correspondencia con el tratamiento térmico recibido.

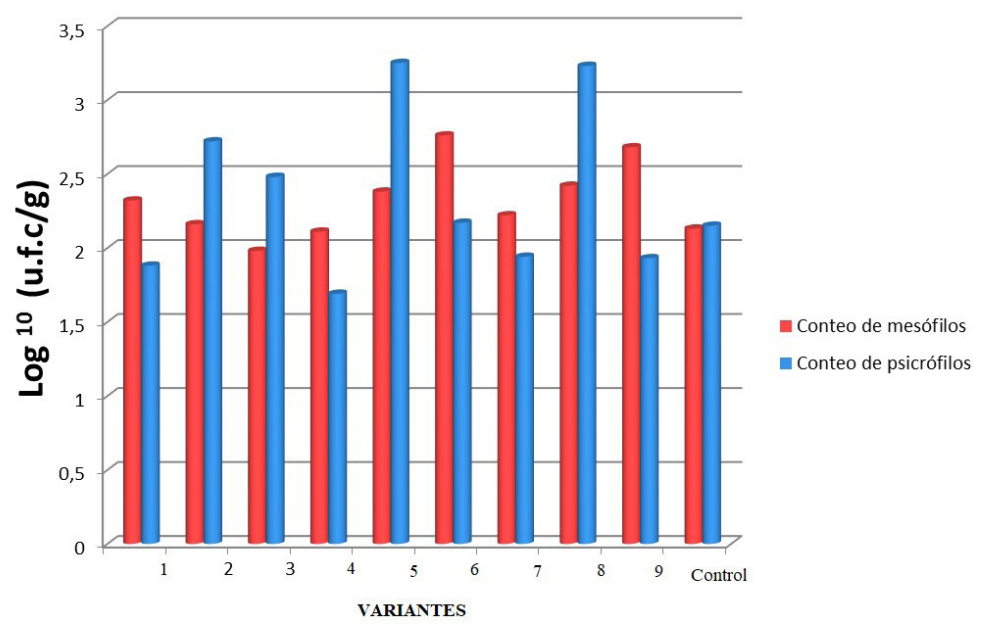

FIGURA 1. Calidad microbiológica inicial de las pastas cárnicas (log UFC/g).

Del análisis de regresión, se pudo observar que los modelos que resultaron significativos $(a=5 \%)$ fueron cuadráticos para el parámetro dureza instrumental y textura sensorial y resultaron no significativos (Tabla 8) para el resto de los parámetros evaluados elasticidad, cohesividad y atributos sensoriales (aspecto, color, sabor y jugosidad). Consecuentemente, no fue posible encontrar para estas variables un modelo matemático que explicara su comportamiento a partir de los niveles estudiados de inulina y grasa. Esto confirmó que dichos parámetros no se vieron afectados con las variaciones realizadas en los factores estudiados.

TABLA 8. Significancia de los modelos de predicción de las variables de respuesta analizadas.

\begin{tabular}{c|c|c|c|c}
\hline Variable & Modelo & $\mathbf{F}$ & $\mathbf{R}^{2}$ & Significancia \\
\hline \multirow{3}{*}{ Dureza } & Lineal & 3,89 & 0,8043 & $\mathrm{~ns}$ \\
\cline { 2 - 5 } & Cuadrático & 44,13 & 0,9692 & $*$ \\
\cline { 2 - 5 } Elasticidad & Lineal & 4,30 & 0,3007 & ns \\
\cline { 2 - 5 } & Cuadrático & 5,27 & 0,5350 & $\mathrm{~ns}$ \\
\cline { 2 - 5 } & &
\end{tabular}




\begin{tabular}{|c|c|c|c|c|c|}
\hline \multirow{13}{*}{$\begin{array}{l}\text { avances } \\
\text { encienciase } \\
\text { ingenierías }\end{array}$} & Variable & Modelo & $F$ & $\mathbf{R}^{2}$ & Significancia \\
\hline & \multirow{2}{*}{ Cohesividad } & Lineal & 7,10 & 0,0051 & ns \\
\hline & & Cuadrático & 11,07 & 0,2051 & ns \\
\hline & \multirow{2}{*}{ Aspecto } & Lineal & 2,68 & 0,2728 & ns \\
\hline & & Cuadrático & 3,78 & 0,4440 & ns \\
\hline & \multirow{2}{*}{ Textura } & Lineal & 42,85 & 0,0248 & ns \\
\hline & & Cuadrático & 6,22 & 0,9153 & * \\
\hline & \multirow{2}{*}{ Sabor } & Lineal & 41,89 & 0,0054 & ns \\
\hline & & Cuadrático & 48,03 & 0,4232 & ns \\
\hline & \multirow{2}{*}{ Color } & Lineal & 1,53 & 0,1409 & ns \\
\hline & & Cuadrático & 1,44 & 0,4571 & ns \\
\hline & \multirow{2}{*}{ Jugosidad } & Lineal & 38,51 & 0,0487 & ns \\
\hline & & Cuadrático & 25,12 & 0,6788 & ns \\
\hline
\end{tabular}

*: Diferencias significativas entre tratamientos al 95\%; ns: diferencias no significativas Los modelos matemáticos de predicción de los parámetros de calidad de las salchichas a partir de las variables contenido de inulina $\left(X_{1}\right)$ y grasa $\left(X_{2}\right)$, cuyos ajustes resultaron significativos, se observan en la Tabla 9.

TABLA 9. Modelos de predicción de los parámetros de calidad de las salchichas

\begin{tabular}{c|c} 
Ecuación & $\mathbf{N}^{\circ}$ \\
\hline Dureza $=5,15+0,23 X_{1}+0,44 X_{2}-0,22 X_{1} X_{2}-0,26 X_{1}^{2}+0,28 X_{2}^{2}$ & Ec. 1 \\
Textura $=5,62+0,055 X_{1}+0,006 X_{2}-0,23 X_{1} X_{2}-0,31 X_{1}^{2}-0,12 X_{2}^{2}$ & Ec. 2
\end{tabular}

$X_{1}$ : contenido de Inulina; $X_{2}$ : contenido de grasa

Los valores de dureza para las variantes estudiadas oscilaron entre 4,50 y 5,85 kg (Tabla 10), correspondiendo el valor más bajo a la variante $8 \%$ de grasa, que contiene en su formulación solo 4\% de almidón de papa. Lo que concuerda con lo descrito por la literatura que señala que en productos donde la disminución del nivel de grasa se realiza aumentando el porcentaje de agua y manteniendo constante la cantidad de proteína, se obtienen productos que exhiben valores inferiores de dureza [6].

TABLA 10. Parámetros texturales de las salchichas elaboradas bajo las diferentes variantes

\begin{tabular}{c|c|c|c|c|c|} 
Variante & Inulina (\%) & $\begin{array}{c}\text { Grasa } \\
(\%)\end{array}$ & $\begin{array}{c}\text { Dureza } \\
\mathbf{( k g )}\end{array}$ & $\begin{array}{c}\text { Elasticidad } \\
\mathbf{( m m )}\end{array}$ & $\begin{array}{c}\text { Cohesividad } \\
\text { (adimensional) }\end{array}$ \\
\hline 1 & 0 & 8 & $4,50(1,15)$ & $6,50(0,03)$ & $0,24(0,02)$ \\
\hline 2 & 6 & 8 & $4,99(0,36)$ & $7,30(0,03)$ & $0,28(0,01)$ \\
\hline 3 & 12 & 8 & $4,97(0,98)$ & $7,50(0,01)$ & $0,25(0,02)$ \\
4 & 0 & 10 & $4,62(0,85)$ & $7,03(0,02)$ & $0,25(0,02)$ \\
5 & 6 & 10 & $5,20(1,34)$ & $7,00(0,01)$ & $0,25(0,02)$ \\
\hline
\end{tabular}




\begin{tabular}{|c|c|c|c|c|c|}
\hline Variante & Inulina (\%) & $\begin{array}{c}\text { Grasa } \\
(\%)\end{array}$ & $\begin{array}{c}\text { Dureza } \\
\text { (kg) }\end{array}$ & $\begin{array}{l}\text { Elasticidad } \\
\qquad(\mathrm{mm})\end{array}$ & $\begin{array}{l}\text { Cohesividad } \\
\text { (adimensional) }\end{array}$ \\
\hline 6 & 12 & 10 & $5,13(0,80)$ & $6,80(0,01)$ & $0,29(0,01)$ \\
\hline 7 & 0 & 12 & $5,43(0,68)$ & $7,35(0,01)$ & $0,28(0,03)$ \\
\hline 8 & 6 & 12 & $5,85(0,83)$ & $7,10(0,01)$ & $0,25(0,01)$ \\
\hline \multirow[t]{2}{*}{9} & 12 & 12 & $5,81(0,67)$ & $7,90(0,08)$ & $0,24(0,02)$ \\
\hline & Control & & $6,60(0,82)$ & $7,00(0,67)$ & $0,25(0,02)$ \\
\hline
\end{tabular}

Como puede observarse en la Figura 2, a medida que aumenta el contenido de inulina y grasa, es mayor la dureza, esto se debe a las propiedades funcionales de la inulina [41] y el almidón de papa [43] las cuales ayudan a retener el agua añadida, formar un gel firme, resistente y proveen cohesividad y estabilidad a la emulsión.

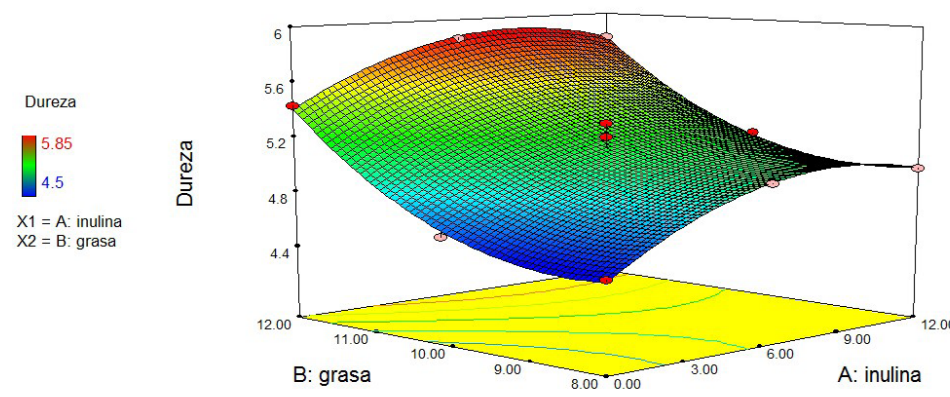

FIGURA 2. Superficie de respuesta para la dureza instrumental $(\mathrm{mm})$ de las salchichas en función del contenido (\%) de inulina y grasa de la formulación.

Los resultados obtenidos son similares a los descritos por diversos autores [46]. En un estudio realizado con salchichas con altos (39\%) y bajos porcentajes en grasa (15\%) y la adición de 7,5\% de inulina, observaron que en salchichas bajas en grasa cuando se adiciona la inulina en forma de polvo, tiende a endurecer el producto. Se puede decir que la grasa enmascara el efecto endurecedor de la inulina en polvo a esa concentración, mientras que la elasticidad fue menor que la del control (39\% de grasa). Cuando la inulina fue añadida en los mismos niveles, pero en forma de gel, no se modificó la elasticidad, pero la cohesividad y dureza mostraron cambios, observándose una menor dureza y cohesividad con respecto al control. Al parecer, la inulina en forma de gel transfiere su característica cremosa a la salchicha volviéndola más suave, alcanzando valores similares de dureza en relación a la salchicha convencional que contiene 39\% de grasa.

En un estudio [44] con salchichas elaboradas con inulina en forma de gel como sustituto de grasa (3 a 12\%), en el que fueron disminuyendo la grasa a medida que incrementaba la concentración de inulina y manteniendo constante el contenido de carne (55\%). Se observó que con el incremento de inulina las salchichas mostraron una vaga tendencia 
a ser más duras, hecho que pudiese ser mayoritariamente atribuido a la reducción del contenido de grasa (de $24 \%$ de grasa en el control a aproximadamente $8,5 \%$ de grasa en las salchichas con 12\% de inulina) que a la adición de inulina. Lo cual concuerda con los resultados de otros investigadores [48,45,12]. No obstante en otro estudio [51], obtuvieron salchichas reducidas en grasa más suaves, donde concentraciones por encima de $10 \%$ de inulina tienen un efecto ablandador significativo, esta discrepancia puede deberse a las diferencias en el diseño experimental y la composición de la inulina, ya que las propiedades de la misma dependen de su grado de polimerización.

Con respecto a la influencia de la grasa y el agua, se conoce que la dureza aumenta al disminuir la grasa, siempre que se mantenga constante el contenido de proteína [10], pero en las pastas cárnicas elaboradas con poca grasa (6 a 12\%) y grandes cantidades de agua, ésta última impide que ocurra un incremento significativo de la dureza, o al menos, evita en algo dicho incremento. En este experimento, en general se aprecia un aumento en los valores de dureza, a medida que se eleva el contenido de inulina, independientemente del nivel de grasa (Tabla 8). Situación que puede deberse a las propiedades funcionales de la inulina y del almidón de papa que ayuda a retener el agua añadida $[10,11,45,12,44]$. La elasticidad no se vio afectada por los porcentajes de inulina y grasa. Estos resultados concuerdan a los encontrados en [12] y [46], quienes trabajaron en mortadela reducida en grasa con adición de inulina en concentraciones del 2,5 al 7,5\% donde obtuvieron valores de elasticidad muy similares con respecto al control en todos los casos.

Aunque la textura sensorial resultó significativa con un nivel de confianza del 95\%, en el análisis de varianza, es importante señalar que al analizar los datos obtenidos de la evaluación sensorial se aprecia que no hay gran variabilidad en los mismos, por lo que, a efecto de la investigación se considera que esta variable respuesta no varía significativamente con los cambios de concentración de inulina y grasa (Figura 3).

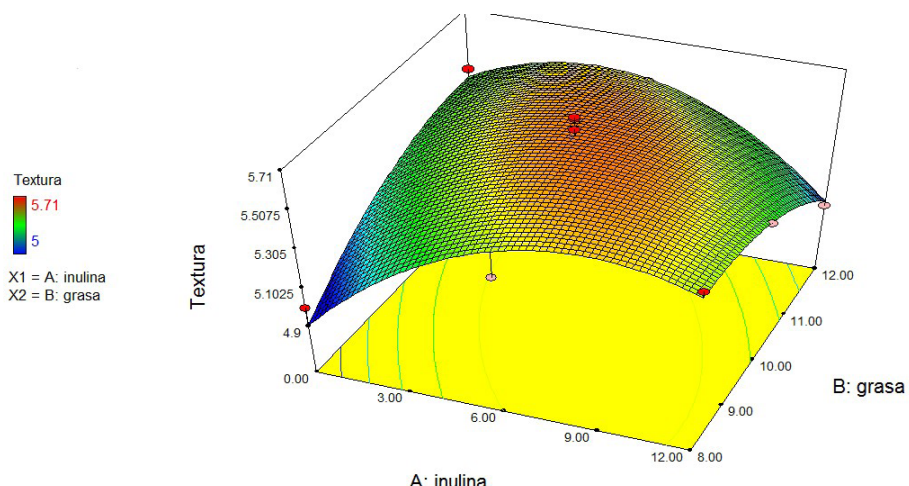

FIGURA 3. Superficie de respuesta de la textura sensorial de las salchichas en función del contenido (\%) de inulina y grasa de la formulación

De la evaluación sensorial los parámetros aspecto, textura, sabor y color, recibieron calificaciones entre 5,0 y 6, es decir de "Bueno" a "Muy Bueno" (Figura 4), lo que significa que estos atributos no se afectaron con la relación inulina-grasa estudiada. A pesar 
de que el atributo jugosidad no resultó significativo, se observa que las variantes con inulina obtuvieron una puntuación mayor que las muestras sin inulina, dado que por sus características funcionales mejora la jugosidad y la sensación bucal de los productos [44]. Las calificaciones de la jugosidad oscilaron entre 3,38 y 4,5, por lo que se podría decir que se obtuvieron productos jugosos. Con lo antes expuesto, se puede evidenciar que estos atributos no fueron afectados en los niveles inulina - grasa ensayados. Puede concluirse, que estos aditivos tienen una importante capacidad para retener la humedad y los jugos de la carne, además de actuar como estabilizadores contribuyendo a la apariencia, palatabilidad y la textura del producto final $[11,41,3]$.

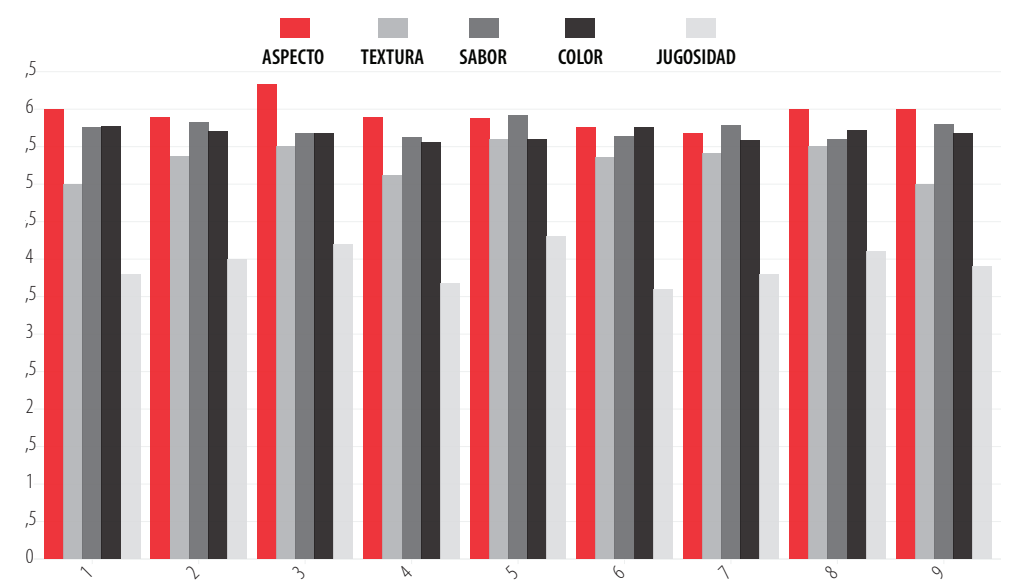

FIGURA 4. Resultados de la evaluación sensorial de las salchichas elaboradas con inulina y almidón de papa

Se destaca que las salchichas con inulina tuvieron un brillo que fue evaluado favorablemente por los panelistas en el parámetro color, no siempre observado en otros tipos de sustitutos de grasa. Este resultado en el color es semejante al reportado en [48] donde se elaboraron salchichas bajas en grasa con incorporación de dos tipos de fibras inulina y oligofructosa al 15\% y 30\%, donde se observó un mejor resultado en cuanto al color en la salchicha con inulina por obtener valores muy parecidos frente al control (con alto porcentaje de grasa).

Con relación al efecto de la inulina y la grasa sobre las puntuaciones otorgadas a la textura, la literatura informa diferentes resultados. $[45,46,52]$ describieron resultados similares a los obtenidos en este experimento, donde los catadores no fueron capaces de detectar los cambios que introdujeron los porcentajes de este aditivo, mientras que otros autores refieren que con cantidades de inulina por encima de $7,5 \%$, los productos recibieron menores calificaciones por los panelistas $[44,12]$.

Resultados similares fueron reportados por [53], quienes estudiaron la influencia de la inulina como sustituto de grasa en bolas de carne de cerdo, evidenciando diferencias significativas en cuanto al color, consistencia, jugosidad, aroma y sabor evaluados sensorialmente frente a un producto control con alto contenido de grasa. Otro trabajo realizado [45], que incorporó inulina a diferentes concentraciones 7,5, 12, 12,5 y 14\%, en salchichas fermentadas reducidas en grasa, describe el mismo efecto en el sabor con calificaciones favorables y sin variabilidad significativa con excepción de la muestra con 
adición de 6\% de inulina en forma de gel que obtuvo una calificación menor. Con estos resultados se confirma lo descrito por [3] que dice que la inulina tiene sabor neutro y no tiene impacto sobre las propiedades sensoriales.

\section{Caracterización del envase}

El material de la tripa está compuesto por poliamida 6, el peso base y espesor fue de 46,23 $\mathrm{g} \cdot \mathrm{m}^{-2}$ y 44,90 $\mu \mathrm{m}$ respectivamente. La fuerza de tensión y la elongación fueron de $70,67 \mathrm{~N} \cdot \mathrm{mm}^{-1}$ y 49,45\% y resistieron adecuadamente la presión de embutido de la salchicha. La permeabilidad al vapor de agua de la tripa fue de 13,15 g. $\mathrm{m}^{-2} \cdot 24 \mathrm{~h}^{-1}$, encontrándose dentro de los valores reportados en la literatura para tripas impermeables [54].

En la Tabla 11 se reporta el resultado obtenido de la medición del espesor del material complejo de ambas caras de la bolsa y sus respectivas capas, así como la identificación de los polímeros que la componen. En dicha tabla se muestra también la permeabilidad al vapor de agua de las dos películas complejas PET/PEBD correspondientes a la cara superior e inferior de la bolsa.

TABLA 11. Características del material de envase en ambas caras de la bolsa

\begin{tabular}{|c|c|c|c|c|}
\hline Material & $\begin{array}{l}\text { Grosor } \\
\text { ( } \mu \mathrm{m})\end{array}$ & $\begin{array}{c}\text { Capa externa } \\
\text { (PET) }\end{array}$ & $\begin{array}{c}\text { Capa } \\
\text { interna } \\
\text { (PEBD) }\end{array}$ & $\begin{array}{c}\text { Permeabilidad vapor } \\
\text { de agua' } \\
\left(\mathrm{g} \cdot \mathrm{m}^{-2} \cdot 24 \mathrm{~h}^{-1}\right)\end{array}$ \\
\hline Cara superior & $89,0(0,8)$ & $22,8(0,4)$ & $66,2(0,6)$ & $1,89(0,06)$ \\
\hline Cara inferior & $88,8(0,8)$ & $22,4(0,5)$ & $66,4(0,5)$ & $2,01(0,07)$ \\
\hline
\end{tabular}

1: Permeabilidad medida a $23^{\circ} \mathrm{C}$ y $85 \%$ HR; PET: Polietileno tereftalato (Poliéster); PEBD: Polietileno de baja densidad; () Desviación Estándar; $n=$

En los resultados de resistencia al sellado térmico de los diferentes cierres de la bolsa se encontró que ninguno de ellos se despegó o partió al aplicárseles tensión. En los cinco ensayos realizados a cada uno de estos cierres, la probeta se elongó más de 25 $\mathrm{mm}$ hasta registrarse una fuerza de tracción de $16 \mathrm{~N}$, partiéndose la película por una zona fuera del área de sellado, lo que indicó la alta resistencia al sellado de estos cierres.

\section{Selección de la mejor variante}

En la Figura 5 se presenta la superficie de respuesta óptima que cumple con las restricciones impuestas en la Tabla 2. Tal como puede apreciarse, hay un área extensa donde se logran fórmulas de óptima calidad. Con el objetivo de obtener un alimento funcional y económico, se selecciona como mejor variante a la de menor contenido de grasa y bajas concentraciones de aditivo: 6,67\% de inulina, $8,73 \%$ de grasa y $4 \%$ de almidón de papa. 


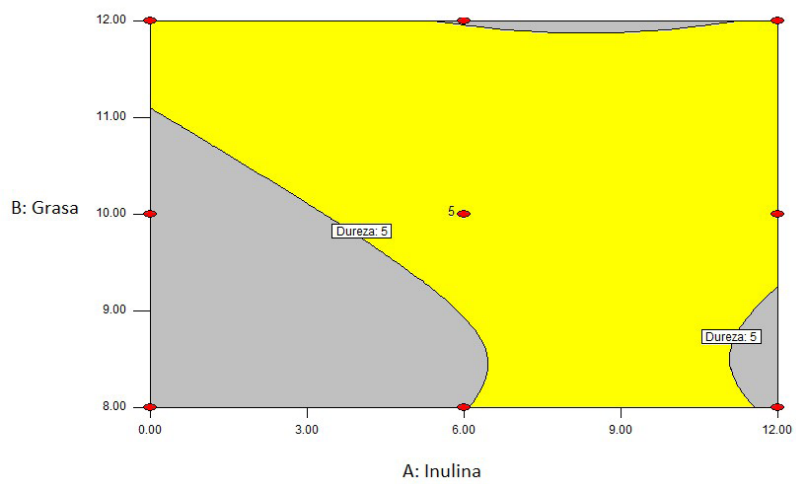

FIGURA 5. Superficie de respuesta óptima de salchichas en función del contenido (\%) de inulina y grasa de la formulación

\section{Vida útil de las salchichas seleccionadas}

Los productos recién elaborados presentaron una composición química acorde con los índices que se prescriben para el mismo (Tabla 12) y se consideraron aptos para realizar las pruebas de vida útil. Los cambios detectados en la humedad indican que la reducción de grasa se hizo a expensas del incremento de la concentración de agua. La actividad de agua $\left(a_{w}\right)$ que es el índice que resulta más importante para la durabilidad, presentó pocas variaciones debido al efecto más fuerte que tiene el cloruro sobre la misma.

TABLA 12. Composición, nitritos pH y aw al inicio del almacenamiento en refrigeración

\begin{tabular}{|c|c|c|c|c|c|c|}
\hline Formulación & $\begin{array}{c}\text { Humedad } \\
(\%)\end{array}$ & $\begin{array}{c}\text { Grasa } \\
(\%)\end{array}$ & $\begin{array}{c}\text { Proteína } \\
(\%)\end{array}$ & $\begin{array}{c}\mathrm{NaNO}_{3} \\
(\mathbf{p p m})\end{array}$ & pH & aw \\
\hline $6,67 \%$ I+8,73\%G & 63,52 & 10,20 & 11,78 & 88,39 & 6,2 & 0,979 \\
\hline
\end{tabular}

Se observó una disminución del pH (Figura 6), más marcado en las muestras no tratadas térmicamente, mientras que en las que se repasteurizaron luego del empacado fue menor. El empacado en bolsas previno a los productos cárnicos de la recontaminación microbiana, que incide en la disminución del pH. A esto contribuyó el tener un nuevo obstáculo, que es la repasteurización, que tiene un efecto reductor sobre la flora contaminante que pudo haber quedado luego del empacado al vacío. 


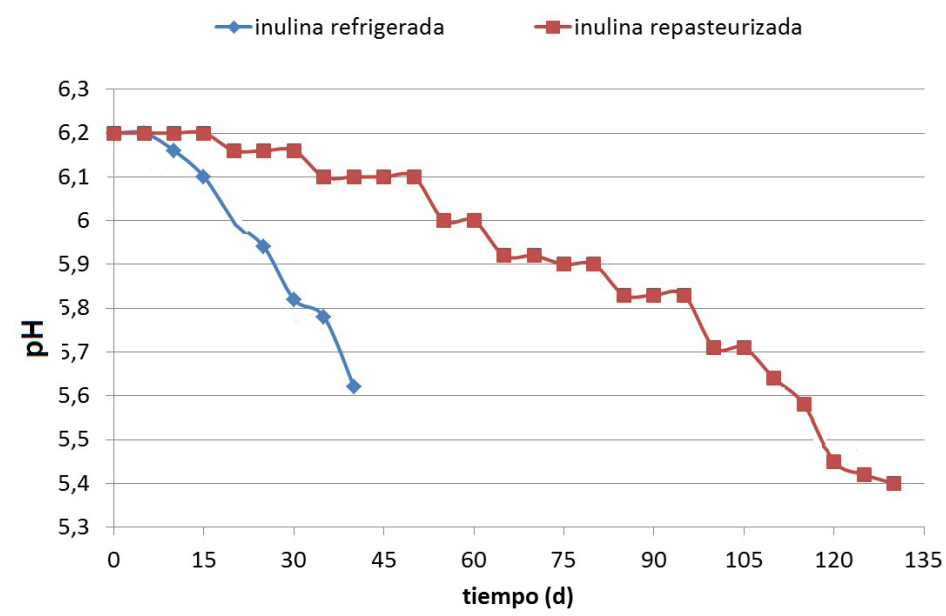

FIGURA 6. Evolución en el tiempo del pH de las salchichas de receta optimizada sometidas a diferentes tratamientos de conservación tras su elaboración.

La disminución de pH estuvo originada por el incremento en los conteos microbianos. Además de la disminución del $\mathrm{pH}$, se vieron afectadas las características organolépticas del producto (olor, sabor y aroma), influyendo directamente en la aceptación del mismo y, por lo tanto, en la vida útil, ya que la evaluación sensorial fue el criterio de rechazo elegido, por ser éste el parámetro utilizado por los consumidores. La calidad microbiológica de los productos al inicio del almacenamiento fue la adecuada, cumpliendo con las exigencias de la Norma de Contaminantes Microbiológicos del Sistema de Normas Sanitarias de Alimentos (Tabla 13).

TABLA 13. Calidad microbiológica de los embutidos tipo salchicha al inicio del almacenamiento en refrigeración.

\begin{tabular}{c|c|c|}
\hline Muestras (t=0) & Aerobios mesófilos (log UFC) & Psicrófilos (log UFC) \\
\hline Con inulina & $1,48 \pm 0,008$ & $2,70 \pm 0,007$
\end{tabular}

El conteo de aerobios mesófilos totales fue del orden de 10²; el de psicrófilos algo más elevado. Es fácil entender esta diferencia entre los conteos pues se trata de un producto refrigerado y el número de enterobacterias fue indetectable (datos no mostrados). Por lo antes expuesto, se puede concluir que los productos recibieron un adecuado tratamiento térmico, siendo elaborados con buenas prácticas de higiene, por lo que fueron aptos para los estudios de durabilidad.

Los conteos microbianos aumentaron significativamente con el tiempo, algo más marcado en las variantes que sólo recibieron el tratamiento de empaque al vacío, mientras que en las repasteurizadas el crecimiento de microorganismos alcanzó la fase de aceleración en un intervalo mayor de tiempo. Esto podría deberse al efecto combinado de los dos obstáculos que deben vencer los microorganismos presentes para desarrollarse. Se observó, además, que en las variantes que sólo fueron empacadas al vacío, en menos de 30 días los conteos de aerobios mesófilos se encontraron en 4 
unidades logarítmicas, valor límite que se permite para este tipo de productos $[55,56]$. En el caso de las variantes que se sometieron a los dos tratamientos (empaque al vacío y repasteurización), después de 110 días, los valores de aerobios mesófilos llegaron al límite establecido.

La tendencia de los conteos de bacterias productoras deácido durante el almacenamiento fue aumentar entre los 35 y 40 días hasta superar las cuatro unidades logarítmicas, dato que coincide con la fecha del rechazo de las variantes empacadas al vacío. Estos microorganismos tienen un lento crecimiento, por lo cual son capaces de desarrollarse en productos envasados al vacío donde otras bacterias de más rápido crecimiento en condiciones aerobias no se desarrollan debido al ambiente microaerofílico.

Al utilizar la repasteurización, el incremento en el tiempo de las bacterias productoras de ácido se hizo más lento y se extendió la durabilidad de los productos, sin embargo, entre los 125 y 130 días los recuentos de estos microorganismos indicadores estuvieron por encima de cuatro unidades logarítmicas. Shumaker y Feirtag (1997) [57] observaron que el deterioro de los productos empacados al vacío ocurría por la presencia de bacterias productoras de ácido que, a pesar de eliminarse durante el tratamiento térmico, se vuelven a reproducir por una recontaminación posterior a la cocción.

Con relación al resto de los indicadores microbianos determinados, en el caso de las levaduras y microorganismos psicrófilos, los conteos durante todo el estudio de durabilidad se mantuvieron en una unidad logarítmica, mientras que no se encontró presencia de coliformes fecales, hongos y Staphylococcus coagulasa positivos, lo que avala la calidad sanitaria de los productos. Además, en ninguno de los productos se encontró presencia de Salmonella (datos no mostrados).

La seguridad y calidad en el estudio estuvieron también relacionadas con las operaciones de envasado, cuyo éxito está en función de las propiedades del material de envase, la eficiencia en conseguir el vacío deseado y la integridad del envase, así como de las condiciones y del control de la temperatura. En las salchichas únicamente envasadas a vacío, se presentó un exudado lechoso y los jueces calificaron el sabor de las mismas como ácidas a partir de los 47 días de almacenamiento.

Los parámetros texturales que se muestran en la Tabla 14, permiten observar que no se encontraron diferencias significativas con un 95\% del nivel de confianza para la fórmula con $5 \%$ de inulina y $8 \%$ de grasa entre el inicio y final del estudio de vida útil de cada formulación.

TABLA 14. Resultados de los parámetros del perfil de textura de las variantes seleccionadas refrigeradas y repasteurizadas-refrigeradas al inicio y final del período de vida útil.

\begin{tabular}{c|c|c|c|c}
\hline Parámetro & Tratamiento & Inicio & Final & Diferencias \\
\cline { 2 - 5 } Dureza $(\mathrm{kg})$ & $\mathbf{C / R}$ & 5,50 & 5,47 & $\mathrm{~ns}$ \\
\cline { 2 - 5 } & $\mathbf{R P} / \mathbf{R}$ & 6,23 & 6,12 & $\mathrm{~ns}$ \\
\cline { 2 - 5 } & $\mathbf{C} / \mathbf{R}$ & 7,32 & 7,28 & $\mathrm{~ns}$ \\
\cline { 2 - 5 } Elasticidad $(\mathrm{mm})$ & $\mathbf{R P} / \mathbf{R}$ & 8,02 & 8,13 & $\mathrm{~ns}$ \\
\cline { 2 - 5 } & &
\end{tabular}




\begin{tabular}{|c|c|c|c|}
\hline $\mathbf{C} / \mathbf{R}$ & 0,23 & 0,22 & ns \\
\hline $\mathbf{R P} / \mathbf{R}$ & 0,25 & 0,23 & ns \\
\hline
\end{tabular}

C/R: Cocinada-refrigerada; RP/R: Repasteurizada-refrigerada; ns: diferencias no significativas al $95 \%$ de confianza

En la Tabla 15 se muestran los resultados del análisis de riesgos de Weibull para la determinación de la vida útil de las salchichas envasadas al vacío y refrigeradas y las que recibieron después de envasadas al vacío un tratamiento de repasteurización y posteriormente se refrigeraron. Se exponen los percentiles del 5\% por ser el riesgo aceptado en el trabajo.

TABLA 15. Vida útil, calculada a partir del análisis de riesgos de Weibull, de las salchichas sometidas a diferentes tratamientos térmicos, y almacenadas en refrigeración entre 2 y $4^{\circ} \mathrm{C}$.

\begin{tabular}{|c|c|c|c|}
\hline \multirow{2}{*}{ Tratamiento } & \multirow{2}{*}{$\begin{array}{c}\text { Promedio } \\
\text { (días) }\end{array}$} & Límite inferior $^{1}$ & Límite superior $^{1}$ \\
\cline { 3 - 4 } & $29,77(0,012)$ & $26,56(0,007)$ & $33,37(0,011)$ \\
\hline C/R & (días) \\
\hline$R P / R$ & $114,37(0,008)$ & $112,18(0,009)$ & $116,60(0,012)$ \\
\hline
\end{tabular}

C/R: Cocinada-refrigerada; RP/R: Repasteurizada-refrigerada; ': promedio +/- intervalo confianza $95 \%$

Seleccionando de estos valores el límite inferior, para una mayor confianza, se puede decir que la vida útil de las salchichas envasadas al vacío y refrigeradas fue de 26 días para la formulación con $6,67 \%$ de inulina, $8,73 \%$ de grasa y $4 \%$ de almidón de papa. Para las salchichas envasadas al vacío y repasteurizadas fue de 112 días para la misma formulación.

\section{CONCLUSIONES}

A partir del presente trabajo de investigación fue posible desarrollar un derivado cárnico emulsificado tipo salchicha con potencialidades funcionales, y que cumplen con el objetivo de reducir el contenido de grasa y una adecuada calidad sensorial y microbiológica, empleando concentraciones entre el 0 y el $12 \%$ de inulina, el $8 \%$ y el $12 \%$ de grasa y un $4 \%$ de almidón de papa, lo que concuerda con lo reportado por otros autores [58]. A partir de la metodología empleada, se verifica que la mejor variante seleccionada para los criterios establecidos dentro de este trabajo correspondió a salchichas elaboradas con un 6,67\% de inulina y un 8,73\% de grasa. Se observó que la inulina produce un aumento significativo de la dureza de los productos, independientemente del nivel utilizado, sin embargo, no afecta las características sensoriales y mantiene la jugosidad de las mismas.

La vida útil de la salchicha con 6,67\% de inulina y $8,73 \%$ de grasa, empacada en bolsas al vacío y almacenada en refrigeración, utilizando como criterio de evaluación el análisis sensorial, se prolongó hasta 26 días, y aplicando un tratamiento adicional de repasteurización, su vida útil llegó a ser de hasta 112 días. Por ello, la técnica de repasteurización puede ser considerada como una opción viable para uso industrial. 


\section{REFERENCIAS}

[1] Alvídrez-Morales, A., González-Martínez, B. E., \& Jiménez-Salas, Z. (2002). Tendencias en la producción de alimentos: alimentos funcionales. Revista salud pública y Nutrición, 3(3).

[2] Lajolo, F.M.; Menezes, E. (2006) Carbohidratos en Alimentos Regionales Iberoamericanos. Ed. Universidad de Sao Paulo. Brasil. p. 549.

[3] Janvary, L. (2005). Inulin, a soluble fibre as fat substitute in meat products. Food Ingredients and Analysis International, 27(4), 25.

[4] Villegas, B. (2008). Efecto de la adición de inulina en las características físicas y sensoriales de batidos lácteos. (Tesis doctoral, Valencia: Universidad Politécnica de Valencia).

[5] Olmedilla-Alonso, Begoña, \& Jiménez-Colmenero, Francisco. (2014). Functional meat products: development and evaluation of their health-promoting properties.Nutrición Hospitalaria, 29(6), 1197-1209. doi: https://dx.doi. org/10.3305/nh.2014.29.6.7389

[6] Jiménez-Colmenero, F. (2004). Estrategias tecnológicas en el desarrollo de derivados cárnicos funcionales. En: F. Jiménez-Colmenero, F. J. Sánchez-Lin, M. J. Y.; Humbert, E. S. y Sosulsky, F. W.n (1974). Journal of Food Science 39(2) 368-370.

[7] Sánchez, A. T. (2007). Productos cárnicos emulsionados bajos en grasa y sodio. Nacameh, 1(1), 53-66.

[8] Colmenero, F. J. (1996). Technologies for developing low-fat meat products. Trends in Food Science \& Technology, 7(2), 41-48. doi: https://doi.org/10.1016/0924-2244(96)81327-6

[9] Pacheco Pérez, W. A., Restrepo Molina, D. A., \& Sepúlveda Valencia, J. U. (2011). Revisión: uso de ingredientes no cárnicos como reemplazantes de grasa en derivados cárnicos. Revista Facultad Nacional de Agronomía Medellín; Vol. 64, núm. 2(2011) 2248-7026 0304-2847.

[10] Ahmed, P. 0., Miller, M. F., Lyon, C. E., Vaughters, H. M., \& Reagan, J. 0. (1990). Physical and sensory characteristics of low-fat fresh pork sausage processed with various levels of added water. Journal of Food Science, 55(3), 625-628. doi: https://doi.org/10.1111/j.1365-2621.1990.tb05192.x

[11] MATULIS, R. J., MCKEITH, F. K., SUTHERLAND, J. W., \& BREWER, M. S. (1995). Sensory characteristics of frankfurters as affected by salt, fat, soy protein, and carrageenan. Journal of Food Science, 60(1), 48-54. doi: https://doi. org/10.1111/j.1365-2621.1995.tb05604.x

[12] Selgas, M. D., Cáceres, E., \& García, M. L. (2005). Long-chain soluble dietary fibre as functional ingredient in cooked meat sausages. Food Science and Technology International, 11(1), 41-47. doi: https://doi. org/10.1177\%2F1082013205051273

[13] Guerra, M., Martín, M., Herrera, H., Jiménez-Colmenero, F., Casals, C. Núñez de Villavicencio, M., Barrero, E. (2001). Estudio de la estabilidad de salchichas con bajo contenido de grasa envasada al vacío. La Industria Cárnica Latinoamericana. №. 124, 33-39.

[14] Jiménez-Colmenero, F. (1995). Productos Cárnicos con bajo contenido en grasa. Eurocarne 35, 53-62.

[15] Mina, J., \& Shirley, K. (2014). Elaboración de salchicha tipo vienesa con sustitución parcial de grasa de cerdo por fibra dietética (Inulina) (Tesis de licenciatura, Machala: Universidad Técnica de Machala).

[16] NC 275. (2005). Norma Cubana. Carne y productos cárnicos. Determinación del contenido de humedad. Método rápido.pp. 6.

[17] NC ISO 1443 (2004). Norma Cubana. Carne y productos cárnicos. Determinación del contenido de grasa total. pp. 6.

[18] NC ISO 937 (2006). Norma Cubana. Carne y productos cárnicos. Determinación del contenido de nitrógeno. pp. 7.

[19] NC ISO 2917 (2004). Norma Cubana. Carne y productos cárnicos. Medición del pH. Método de referencia. pp. 9.

[20] NC ISO 712. (2002). Norma Cubana. Cereales y productos de cereales. Determinación del contenido de humedad. pp. 11.

[21] NC ISO 2171 (2002). Norma Cubana. Cereales y productos de cereales. Determinación de cenizas. pp. 9. 
[22] Horwitz, W. (1975). Official methods of analysis (Vol. 222). Washington, DC: Association of Official Analytical Chemists.

[23] Imeson, A. (Ed.). (2011). Food stabilisers, thickeners and gelling agents. John Wiley \& Sons.

[24] Bourne, M. C. (1978). Texture profile analysis. Food Technology. 32(7): 62-66, 72.

[25] NC ISO 936. (2006). Norma Cubana. Carne y productos cárnicos. Determinación de ceniza total. pp. 9.

[26] NC ISO 1841-1 (2004). Norma Cubana. Carne y productos cárnicos. Determinación del contenido de cloruro. Parte l: Método de Volhard. pp. 8.

[27] NC 357 (2004). Norma Cubana. Carne y productos cárnicos. Determinación del contenido de nitrito. pp. 6.

[28] NC ISO 4833-1 (2014). Norma Cubana. Microbiología de la cadena alimentaria. Método horizontal para la enumeración de microrganismos. Parte 1: Conteo de colonias a $30^{\circ} \mathrm{C}$ por la técnica de placa vertida. pp. 12.

[29] NC ISO 4831 (2010). Norma Cubana. Microbiología de alimentos de consumo humano y animal. Método horizontal para la detección y enumeración de coliformes. Técnica del número más probable. pp.14.

[30] NC ISO 4832 (2010). Norma Cubana. Microbiología de alimentos de consumo humano y animal. Método horizontal para la detección y enumeración de coliformes. Técnica de conteo de colonias método de referencia. pp. 10.

[31] NC 1004 (2014). Microbiología de alimentos de consumo humano y animal. Guía general para la enumeración de levaduras y mohos. Técnica a $25^{\circ} \mathrm{C}$. pp. 11.

[32] NC ISO 6579 (2008). Norma Cubana. Microbiología de alimentos de consumo humano y animal. Método horizontal para la detección de Salmonella spp. Método de referencia. pp. 36.

[33] NC IS0 6888-1 (2003). Norma Cubana. Microbiología de alimentos de consumo humano y animal. Método horizontal para la enumeración de Staphylococcus coagulasa positiva (Staphylococcus aureus y otras especies). Parte 1: Técnica utilizando el medio agar Baird Parker. pp. 17.

[34] NC IS0 536 (1999). Norma Cubana. Papel y cartón. Determinación del gramaje. pp. 8.

[35] ASTM E252-06 (2013). Standard Test Method for Thickness of Foil, Thin Sheet, and Film by Mass Measurement. ASTM International, West Conshohocken, PA. www.astm.org

[36] NC IS0 527-3 (2012). Norma Cubana. Plásticos. Determinación de las propiedades en tracción. Parte 3: Condiciones de ensayo para películas y hojas. pp. 7.

[37] NC ISO 2528 (2010). Norma Cubana. Materiales en lámina. Determinación de la velocidad de transmisión al vapor de agua. Método gravimétrico (de la cápsula). pp. 18.

[38] ASTM F88/F88M-09 (2009). Standard Test Method for Seal Strength of Flexible Barrier Materials. ASTM International, West Conshohocken, PA. www.astm.org

[39] Guerra, M. A., Pérez, D., Hernández, U., de Hombre Morgado, R., Frómeta, Z., Pérez, G., \& Rodríguez, F. (2017). Efecto de la harina de plátano sobre la calidad de un embutido tipo mortadella. Ciencia y Tecnología de Alimentos, 21(3). pág. 22-26.

[40] Cardelli, C., \& Labuza, T. P. (2001). Application of Weibull hazard analysis to the determination of the shelf life of roasted and ground coffee. LWT-Food Science and Technology, 34(5), 273-278. doi: https://doi.org/10.1006/ fstl.2000.0732

[41] Madrigal, L., \& Sangronis, E. (2007). La inulinay derivados como ingredientes claves en alimentos funcionales. Archivos latinoamericanos de nutrición, 57(4), 387-396.

[42] Phillips, G. 0., \& Williams, P. A. (Eds.). (2000). Handbook of hydrocolloids (pp. 53-64). Boca Raton, FL: CRC press.

[43] Carballo, J., Barreto, G., \& Colmenero, F. J. (1995). Starch and egg white Influence on properties of bologna sausage as related to fat content. Journal of Food Science, 60(4), 673-677. doi: https://doi.org/10.1111/j.1365-2621.1995. tb06204.x

[44] Nowak, B., Von Mueffling, T., Grotheer, J., Klein, G., \& Watkinson, B. M. (2007). Energy Content, Sensory Properties, and Microbiological Shelf Life of German Bologna-Type Sausages Produced with Citrate or Phosphate and with Inulin as Fat Replacer. Journal of Food Science, 72(9), S629-S638. doi: https://doi.org/10.1111/j.1750-3841.2007.00566.x 
[45] Mendoza, E., García, M. L., Casas, C., \& Selgas, M. D. (2001). Inulin as fat substitute in low fat, dry fermented sausages. Meat science, 57(4), 387-393. doi: https://doi.org/10.1016/50309-1740(00)00116-9

[46] Luisa García, M., Cáceres, E., \& Dolores Selgas, M. (2006). Effect of inulin on the textural and sensory properties of mortadella, a Spanish cooked meat product. International journal of food science \& technology, 41(10), 1207-1215. doi: https://doi.org/10.1111/j.1365-2621.2006.01186.x

[47] Mencía Guevara, A. M., Fuentes, B., \& Acosta Marchetti, A. (2010). Efecto de dos concentraciones de fosfato, de grasa y la adición de inulina sobre las propiedades físicas y sensoriales de una salchicha de pollo reducida en sal (№. T3028). ESCUELA AGRÍCOLA PANAMERICANA,.

[48] Villalobos, C. E. V., Simental, S. S., \& Delgado, L. H. V. (2010). Efecto de la fibra dietética sobre la textura de salchichas tipo Viena. Nacameh, 4(2), 37-43.

[49] Mittal, G. S., \& Barbut, S. (1994). Effects of fat reduction on frankfurters' physical and sensory characteristics. Food Research International, 27(5), 425-431. doi: https://doi.org/10.1016/0963-9969(94)90236-4

[50] NTE INEN 1338 (2012). Norma Técnica Ecuatoriana. Carne y productos cárnicos. Productos cárnicos crudos, productos cárnicos curados-madurados y productos cárnicos precocidos-cocidos. Requisitos. pp. 11.

[51] Cáceres, E., Garcıa, M. L., Toro, J., \& Selgas, M. D. (2004). The effect of fructooligosaccharides on the sensory characteristics of cooked sausages. Meat Science, 68(1), 87-96. doi: https://doi.org/10.1016/j.meatsci.2004.02.008

[52] Hadorn, R., Piccinali, P., Guggisberg, D., \& Suter, M. (2008). Inulin-induced fat reduction in lyoner sausages. In 54th International Congress of Meat Science and Technology (Vol. 3, pp. 1-3).

[53] Flaczyk, E., Górecka, D., Kobus, J., \& Szymandera-Buszka, K. (2009). The influence of inulin addition as fat substitute on reducing energy value and consumer acceptance of model pork meatballs. Żywność Nauka Technologia Jakość, 16(4).

[54] Theller, H. W. (1989). Heatsealability of flexible web materials in hot-bar sealing applications. Journal of Plastic Film \& Sheeting, 5(1), 66-93. doi: https://doi.org/10.1177\%2F875608798900500107

[55] ICMSF (1986). Ecología Microbiana de los Alimentos. Acribia, Zaragoza.

[56] NC 585 (2015). Norma Cubana. Contaminantes microbiológicos en alimentos. Requisitos sanitarios (Obligatoria). pp. 27.

[57] Shumaker, S., \& Feirtag, J. (1997). Environmental analysis methods utilized to determine the contamination sources in a sausage processing plant. Dairy, food and environmental sanitation, 17(5), 274-280.

[58] Chávez, N.A.; Rodríguez, G.M. (2017). Elaboración de un producto cárnico funcional. (Tesis de doctorado, Universidad Autónoma de Aguas (alientes). 